\title{
CORRECTION
}

Hugh J. A. Bird $[$ Kiran Ramesh $(1)$

\section{Correction to: Unsteady lifting-line theory and the influence of wake vorticity on aerodynamic loads}

Published online: 3 September 2021

(C) Springer-Verlag GmbH Germany, part of Springer Nature 2021

\section{Correction to: Theor. Comput. Fluid Dyn. \\ https://doi.org/10.1007/s00162-021-00578-8}

The Original publication of this article was published with distorted Figure 7. The correct Fig. 7 is given below. The original article has been corrected.

The original article can be found online at https://doi.org/10.1007/s00162-021-00578-8

H. J. A. Bird · K. Ramesh $(\varangle)$

Aerospace Sciences Division, School of Engineering, University of Glasgow, Glasgow G12 8QQ, UK

E-mail: kiran.ramesh@glasgow.ac.uk 

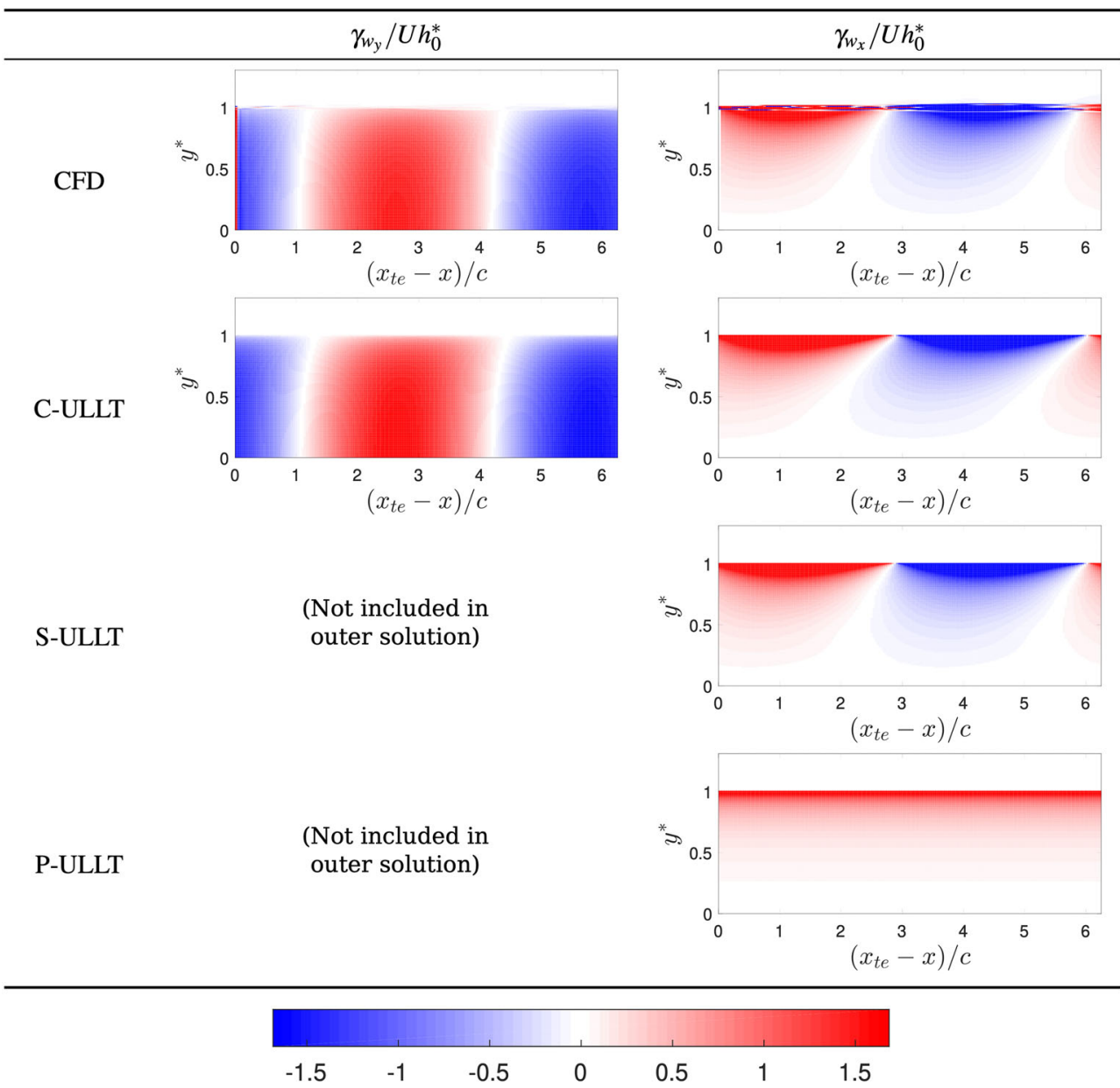

Fig. 7 Comparison of wake vortex sheet strength for an aspect ratio 4 wing oscillating at $k=0.5$. The ULLT results show only the wake model assumed in the outer domain. Since the wing is shrunk to a line in the outer domain of the ULLTs, the $x$ coordinate of the trailing edge, $x_{\mathrm{te}}$, is zero for ULLTs. Shown at $t \bmod 2 \pi / \omega=0$

Publisher's Note Springer Nature remains neutral with regard to jurisdictional claims in published maps and institutional affiliations. 